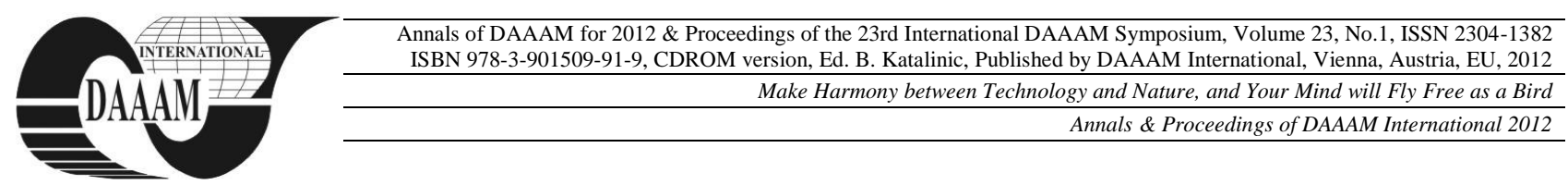

\title{
SENSITIVITY OF SUBPIXEL CORNER DETECTION
}

\author{
SROBA, L[ukas] \& RAVAS, R[udolf]
}

\begin{abstract}
The corner point detection is widely used in image processing area and is very important in many practical applications. But sometimes this pixel accuracy is not enough. In that case we can use some mathematical techniques and try to find a corner point in subpixel accuracy. We have described principles of 5 subpixel detectors in this paper. The Harris corner detector was chosen for our test of subpixel detection sensitivity. For that reason we used pointing device having accuracy up to 0.01 pixels. We tested what is the smallest shift of testing image, which can be recognized by detector. There were the appropriate statistical analysis performed and the results were illustrated in graphs and table. The sensitivity of detection was determined for single image and for sequence of images too.
\end{abstract}

Keywords: subpixel detection, sensitivity of detectors, X-corner point, Harris detector

\section{INTRODUCTION}

As it was said, the corner detection is very important in image pre-processing. Practical using could be motion tracking, object detection and recognition, 3D modeling, robot navigation, stitching of panoramic photographs and stereo matching for example. There is no exact definition what the corner point is, but we can use the following statements: Corner point may be the point where at least two edged are intersected, point having the smallest radius of curvature or point around which is high change of brightness intensity in all directions.

As it is known, the smallest part of an image is a pixel. We can't access information "between" pixels in usual. But there is a possibility to use mathematical techniques to interpolate the brightness intensity between pixels and increase the accuracy of detected corner points. It could be convenient to camera calibration task. This subpixel detection can also decrease the costs of camera, because we don't need to buy the high-resolution camera and we can use simpler camera for that purpose in some cases.

References [1] and [2] deal with precision of subpixel detection for example.

\section{SUBPIXEL DETECTORS}

There are 5 subpixel detectors presented in this part. Two of them - Harris corner detector and Haralick interest operator are well known around the world and often used in practical fields. That's the reason why we chose Harris corner detector for our tests of sensitivity. The rest 3 of them are described just for information and they aren't suitable for real applications as good as the previous two [3].

\subsection{Harris corner detector}

The Harris corner detector is widely used in many real applications and can detect corner point not only in pixel accuracy, but in subpixel accuracy too. This detector was developed by Chris Harris and Mike Stephens in 1988 [4]. The basic idea is to find the minimum of intensity difference between a chosen part of an image (marked as $W$ ) and shifted part of image $W$ in all directions. There is Taylor series approximation for that purpose used.

First step is calculation of matrix $A$. The variables $I_{x}$ and $I_{y}$ are approximations of derivations (also referred as differences) in horizontal and vertical direction. These differences are usually computed by using suitable convolution masks. The window $W$ has usually size $5 \times 5$ or $7 \times 7$ pixels. The matrix $A$ is calculated for every pixel in image by following formula:

$$
A=\sum_{W}\left(\left[\begin{array}{l}
I_{x} \\
I_{y}
\end{array}\right] \cdot\left[\begin{array}{ll}
I_{x} & I_{y}
\end{array}\right]\right)
$$

Next step is determination of matrix $C$ by using (4). The matrix $C$ has the same size as original image and contains the specific value for every pixel in image. The components $\operatorname{det}(A)$ and trace $(A)$ are computed from matrix $A$ for every pixel. The variable $k$ has usually the value between 0.04 and 0.06 .

$$
\begin{gathered}
\operatorname{det}(A)=A(1,1) \cdot A(2,2)-A(1,2)^{2} \\
\operatorname{trace}(A)=A(1,1)+A(2,2) \\
C=\operatorname{det}(A)-k \cdot \operatorname{trace}(A)
\end{gathered}
$$

Last step is searching the elements in matrix $C$ having highest values. These points can be stated as corner points. It is necessary to use global or local thresholding of course.

As we can see, this procedure can help us to find the corner points in pixel accuracy. This part of algorithm could be the same for every subpixel detector. We find pixel coordinates of corner points, choose suitable window $W$ surrounding chosen pixel and then we use specific subpixel detector for obtain the more precise subpixel coordinates. It is not obligatory to use only Harris detector for pixel coordinates of corner points.

If we want to obtain the subpixel coordinates using Harris detector, we need to interpolate the brightness 
intensity between the pixels. For that reason bilinear or bicubic interpolation is usually used. The procedure is following: Firstly we find corner points in pixel accuracy according the method we mentioned before. Then we choose the small window surrounding corner pixel and define the brightness intensity values between pixels using interpolation. We may use step 0.01 pixel between original pixels for example. It means that 1 pixel contains 100 subpixels in this case. So we can reach 100 times higher accuracy as initially. The rest of the procedure is exactly the same as we described before.

\subsection{Haralick interest operator}

The Haralick interest operator was originally described in [5]. Firstly this detector selects the window of interest $W$ and afterwards computes more precisely the subpixel position of corner point inside of this window according (5). Differences $I_{x}, I_{y}$ and their squares $I_{x}{ }^{2}, I_{y}{ }^{2}$ are computed by using convolution masks.

$$
\begin{aligned}
& \left(\begin{array}{cc}
\sum_{W} I_{y}^{2} & \sum_{W} I_{x} \cdot I_{y} \\
I_{x} \cdot I_{y} & \sum_{W} I_{x}^{2}
\end{array}\right)\left(\begin{array}{l}
y_{c} \\
x_{c}
\end{array}\right)= \\
& =\left(\begin{array}{c}
\sum_{W}\left(y \cdot I_{y}^{2}+x \cdot I_{x} \cdot I_{y}\right) \\
\sum_{W}\left(x \cdot I_{x}^{2}+y \cdot I_{x} \cdot I_{y}\right)
\end{array}\right)
\end{aligned}
$$

We get the subpixel coordinates $y_{c}$ and $x_{c}$ after we solve this system of 2 equations. But it is important to notice, that the whole theory about this detector is much more sophisticated.

These two mentioned subpixel detectors can find any kind of regular corner point. The following detectors can only find subpixel coordinates if we consider X-corner points. X-corner points are easy to imagine like parts of chessboard image.

\subsection{Subpixel detector based on saddle points}

This algorithm for detecting X-corner points has a different approach in compare to previous ones and we don't need to compute any differences or derivates. This approach was described in [6]. For every detected Xjunction a quadratic fitting of the brightness intensity profile is obtained by solving (6). This formula describes the geometrical structure called hyperbolic paraboloid whose critical points are often referred as saddle points. These saddle points represent the exact position of $\mathrm{X}$ corner points.

$$
h(x, y)=a x^{2}+b x y+c y^{2}+d x+e y+f
$$

As you can see, at least 6 values of brightness intensity are necessary to get the solution. We take these values from inside the chosen window $W$. It gives us a system of 6 equations and it can be solved by using the least squares method for example. It is possible to use overdetermined system of course.

$$
\left(\begin{array}{l}
x \\
y
\end{array}\right)=-\left(\begin{array}{cc}
2 a & b \\
b & 2 c
\end{array}\right)^{-1} \cdot\left(\begin{array}{l}
d \\
e
\end{array}\right)
$$

If the variables $a, b, c, d, e$ and $f$ are calculated, we use (7) to get the final subpixel coordinates of X-corner point.

\subsection{Subpixel detector based on iterations}

This algorithm is described in [7] and is based on Orthogonal vectors theory. The basic presumption is that the vector from X-corner (marked $q$ ) to any part of its adjacent area (marked $p$ ) should be perpendicular to the gradient of point $p$. It is mathematically written in (8). $I\left(p_{i}\right)$ is gradient in point $p$ and is composed of $I_{x}$ and $I_{y}$.

$$
I\left(p_{i}\right)^{T} \cdot\left(q-p_{i}\right)=0
$$

If we modify this equation, multiply both sides by $I\left(p_{i}\right)$ and use the points from the adjacent area $W$, the formula looks like (9).

$$
\left.\sum_{W}\left(I\left(p_{i}\right) \cdot I\left(p_{i}\right)^{T}\right) \cdot q=\sum_{W} I\left(p_{i}\right) \cdot I\left(p_{i}\right)^{T} \cdot p_{i}\right)
$$

After solving this equation and computing $q$, the new point $q$ is taken as the new center of the searching window $W$ and we solve the equation through the iterations. We can choose the fix number of iterations or some other terminal conditions.

\subsection{Subpixel detector based on Taylor series}

This way how to obtain the subpixel position of $\mathrm{X}$ corner point is mentioned in [8]. It is obvious that the real subpixel position of pixel accuracy corner point must be located in its adjacent area. We can use a second Taylor polynomial to describe the local intensity profile around X-corner. The values of shifts $\Delta_{x}$ and $\Delta_{y}$ can be computed by using (10) and (11). The signs $I_{x}, I_{v}$ are first differences (as simplification of first derivatives) and $I_{x x}$, $I_{v v}, I_{x v}$ means second differences (second derivatives) in corresponding directions.

$$
\begin{aligned}
& \Delta_{x}=\frac{I_{y} I_{x y}-I_{x} I_{y y}}{I_{x x} I_{y y}-I_{x y}^{2}} \\
& \Delta_{y}=\frac{I_{x} I_{x y}-I_{y} I_{x x}}{I_{x x} I_{y y}-I_{x y}^{2}}
\end{aligned}
$$

These two formulas are based on assumption that the $\mathrm{X}$-corner point is the saddle point of intensity profile. If we set the first order partial derivatives of second Taylor polynomial to zero, we are able to find this saddle point and define $\Delta_{x}$ and $\Delta_{y}$.

\section{EXPERIMENTAL TESTS}

The main goal of this paper is to determine the sensitivity of Harris corner detector. For that reason we chose very accurate pointing device [9], as it is illustrated in fig. 1. We fixed a small picture of chessboard segment to pointing device and we set small shifts on device (the orders of hundredths of millimeter). Then we took series of images with high-resolution camera and we found subpixel coordinates ( $x$ axis is sufficient because of movement in horizontal direction) for every single image from series. There were taken 30 images for position $1-$ 4 and 20 images for position $5-6$ (6 positions in total) in measurement 1 . Measurement 2 consists of 30 images for every position (10 positions in total). We performed statistical analysis of our results and created appropriate graphs and table. All shifts on pointing device are recalculated in pixels, not hundredths of millimeters.

It's important to notice, that we got the images in standard lighting conditions. For that reason, there is an influence of brightness intensity changes. 


\section{EXPERIMENTAL RESULTS}

There are 4 types of values shown in fig. 2 and fig. 3 (marked as $A, B, C$ and $D$ in legend). The sign $A$ is arithmetic mean of all values we obtained for one position (one shift) of pointing device. The $B$ means maximal and minimal values of found coordinates for every single position. The sign $C$ represents the boundaries of $3 \sigma$ intervals around arithmetic mean and $D$ describes boundaries of $3 \sigma$ intervals calculated specifically for arithmetic mean.

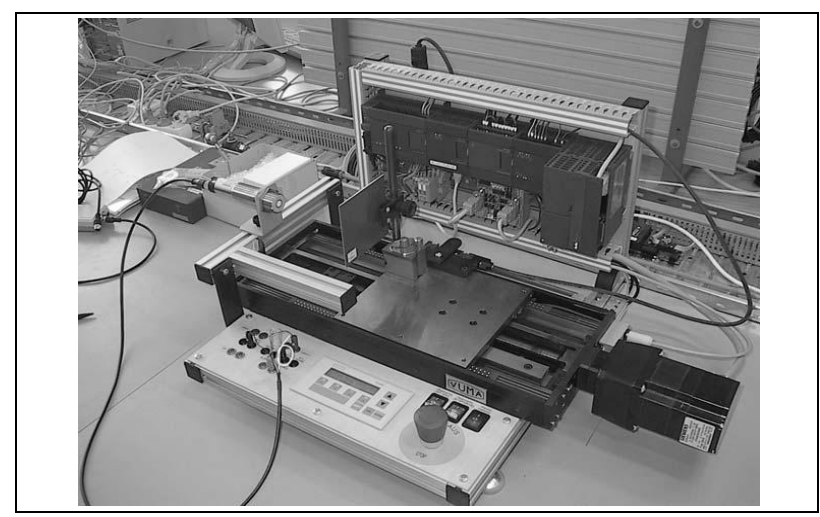

Fig. 1. The chosen pointing device

This kind of standard deviation for arithmetic mean is defined according to (12):

$$
\sigma=\sqrt{\frac{\sum_{i=1}^{N}\left(x_{i}-\mu\right)^{2}}{N(N-1)}}
$$

$N$ is number of all elements, $x_{i}$ is current element and $\mu$ means arithmetic mean. The $x$ axis consists of shifts on pointing device stated in pixels and $y$ axis contains horizontal positions of corner point.

If we compare $3 \sigma$ borders between various positions in fig. 2 or fig. 3 , we can determine the smallest distance in pixels, which is necessary to differentiate shift of image.

As we can see, the distance around 15 hundredths of pixel is enough for our purpose. It means, that if we take one image, shift the chessboard picture in 15 hundredths of pixel (or recalculate it to fractions of millimeters) and take another image, Harris subpixel detector can recognize the difference if we consider the rule of confidence interval $3 \sigma$. It means, we were looking for the distance between two positions where there is no intersection of their $3 \sigma$ intervals (it gives us 99.73 percent chance of right decision for normal distribution).

In the case, we take a set of photos and we calculate the arithmetic mean of all obtained subpixel coordinates, we should consider the confidence interval $3 \sigma$ for arithmetic mean. It is possible to see, that difference around 3 hundredths of pixel between photos is sufficient to Harris detector.

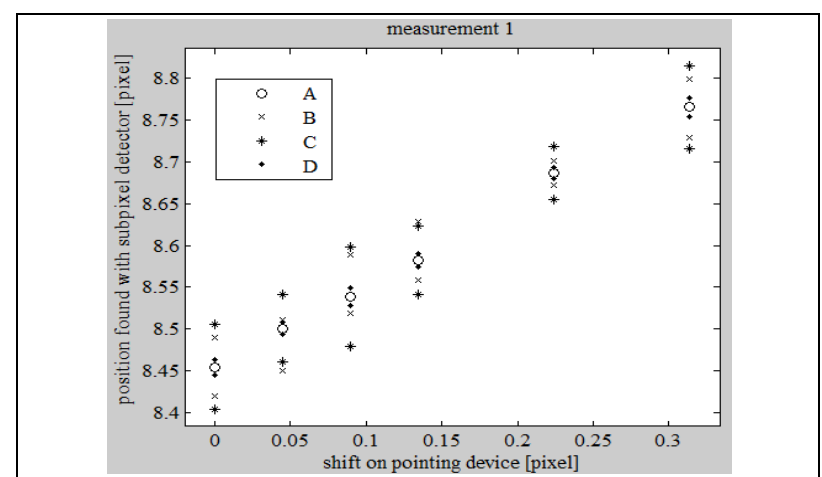

Fig. 2. Statistical analysis of horizontal coordinate for every position

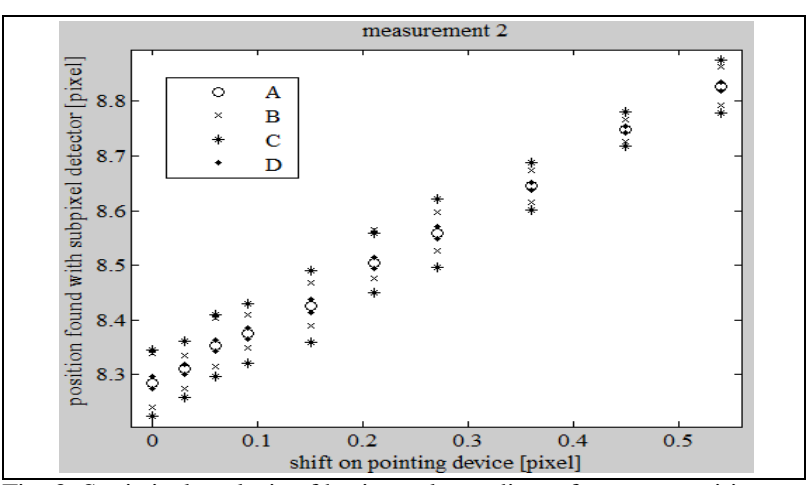

Fig. 3. Statistical analysis of horizontal coordinate for every position

Fig. 4 and fig. 5 deal with difference between obtained (stated arithmetical mean) and theoretical position of corner point. It's important to notice, that we didn't use the perfectly calibrated camera and we didn't know the exact focal length of camera. For that reason, we matched the last obtained and last theoretical coordinate to the same place in graphs and we multiply found coordinates for every position by given correction coefficient.

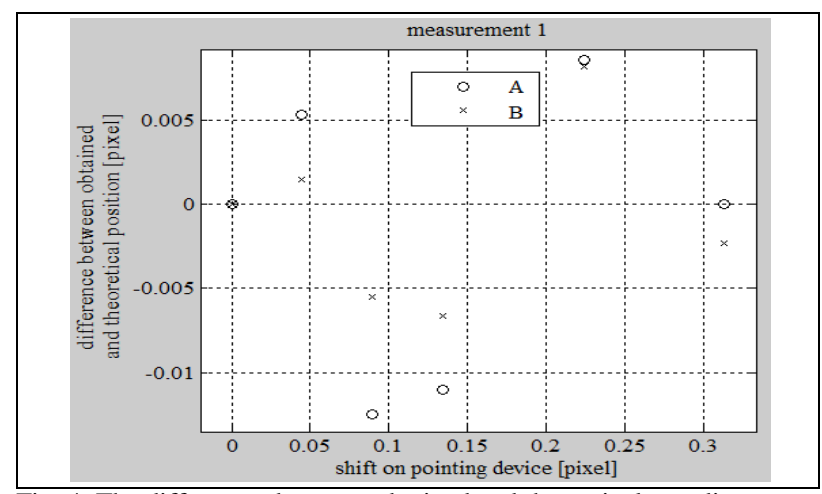

Fig. 4. The differences between obtained and theoretical coordinates

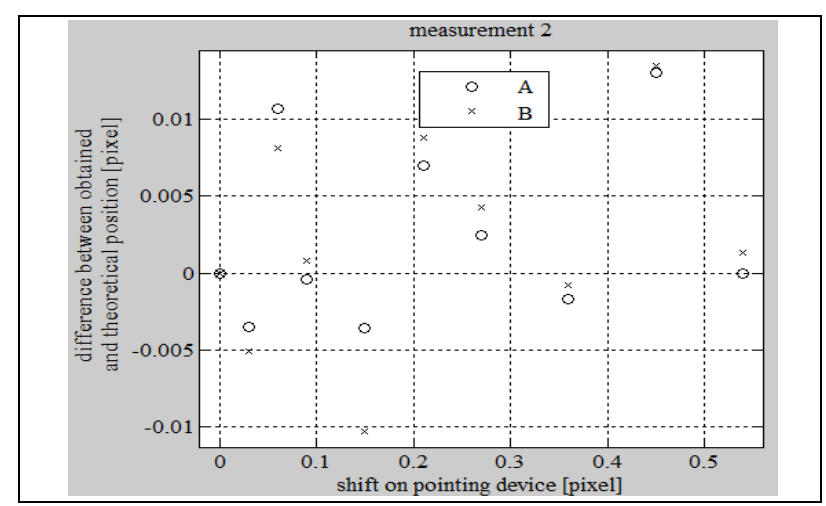

Fig. 5. The differences between obtained and theoretical coordinates 
The $x$ axis is analogous to previous graphs and $y$ axis contains differences between coordinates we got and theoretical values of coordinates. The sign $A$ represents trimmed means and it is calculated from truncated values. It means, that extreme values from set of given coordinates were neglected. The $B$ is determined from full data set, what means we consider the regular set of coordinates for our analyses.

As it is obvious from graphs, the maximal difference between obtained and theoretical coordinates is about 13 thousandths of pixel.

There are listed the standard deviations $\sigma$ for all shifts in tab. 1. As it is possible to see, the average standard deviation for whole measurement is around 15-18 thousandths of pixel. There is influence of brightness intensity changes as the main factor of these deviations.

\begin{tabular}{|r|r|r|r|}
\hline measurement 1 & & measurement 2 & \\
\hline shift [pixel] & $\sigma$ [pixel] & shift [pixel] & $\sigma$ [pixel] \\
\hline 0 & 0,0170 & 0 & 0,0200 \\
\hline 0,0488 & 0,0135 & 0,0300 & 0,0174 \\
\hline 0,0896 & 0,0197 & 0,0600 & 0,0187 \\
\hline 0,1344 & 0,0138 & 0,0900 & 0,0180 \\
\hline 0,2240 & 0,0105 & 0,1500 & 0,0221 \\
\hline 0,3136 & 0,0166 & 0,2100 & 0,0180 \\
\hline & 0,0152 & 0,2700 & 0,0208 \\
\hline & & 0,3600 & 0,0144 \\
\hline \multirow{2}{*}{ average $\sigma$} & & 0,4500 & 0,0105 \\
\cline { 3 - 4 } & & 0,5400 & 0,0159 \\
\cline { 3 - 4 } & & average $\sigma$ & 0,0176 \\
\hline
\end{tabular}

Tab. 1. The standard deviations for every shift in both measurements

\section{CONCLUSIONS}

This paper has dealt with chosen subpixel corner detectors and sensitivity of subpixel corner detection. We have decided to test Harris detector because of its very good detection qualities and possibility to be successfully applied in real conditions.

We implemented our own experiments where we chose pointing device having shift accuracy up to 0.01 pixels. Then we were changing position (various shifts) of chessboard image and we were observing how our obtained coordinates are changing. We performed statistic analyses of given data and there were corresponding graphs and table created. For these tasks there were appropriate computational scripts in development environment Matlab created.

As it is shown in fig. 2 and fig. 3, if we shift an image in around 0.15 pixels, the detector can recognize the difference on confidence interval $3 \sigma$. If we take series of images, we can determine the sensitivity for arithmetic mean of found positions. We found that shift around 0.03 pixels is sufficient in this case. The basic assumption is that if we consider the arithmetic mean and we take $n$ images, the sensitivity of detector can be decreased by a square root of $n$. It is important to notice, that these results are influenced by brightness intensity conditions.

Fig. 4 and fig. 5 explain how can be difference between obtained and theoretical position. We stated the comparison for both truncated and regular data. We found out that the maximal difference is about 0.13 pixels. This difference is caused by variation of light conditions during the experiments.
We stated standard deviations of obtained coordinates from set of taken images for every position in both measurements. These results are listed in tab. 1 . In our case, these values are in interval $0.015-0.018$ pixels.

And last but not least we demonstrated how the light conditions are important in subpixel detection. We can see that only one factor influencing and modifying our results is brightness intensity change in testing images.

There are a few limitations in our approach of course. Firstly, the camera has limited resolution and limited bit depth. The pointing device is not absolutely precise and we are not able to take infinity number of images. Because of that our statistic analyses is not as accurate as it could be. Take more than one image is reasonable only for static scene, in case we have dynamic scene there are other issues we need to consider.

There are many ways how to continue to this research. One of them is to calculate the average value of brightness intensity for the whole set of photos in every particular pixel and then use subpixel detector. It's our plan to publish these results in future too. Another research may be oriented to use a camera having higher values of bit depth parameter. It could be interesting to observe how increasing of brightness image quantization can improve the accuracy of subpixel detection.

\section{ACKNOWLEDGEMENT}

This work was supported by the Slovak Research and Development Agency under the contract No. APVV0333-11. Special thanks to Ing. Martin Juhas, $\mathrm{PhD}$. from Institute of automation, measurement and applied informatics in Faculty of Mechanical Engineering (Slovak University of Technology in Bratislava) for possibility to use their equipment and devices.

\section{REFERENCES}

[1] Stock, Ch., Muhlmann, U., Chandraker, M. K. \& Pinz, A. (2002). Subpixel Corner Detection for Tracking Applications using CMOS Camera Technology, 26 ${ }^{\text {th }}$ Workshop of the AAPR/OAGM, pp. 191-199

[2] Cigada, A., Sala, R. \& Zappa, E. (2003). On the Reduction of Sub-pixel Error in Image Based Displacement Measurement, XVII IMEKO World Congress Metrology in $3^{\text {rd }}$ Millenium, pp. 1947-1951

[3] Sroba, L. \& Ravas, R. (2012). The subpixel X-corner detectors and their window shift robustness comparison, ELITECH'12. $14^{\text {th }}$ Conference of Doctoral Students, ISBN 978-80-227-3705-0

[4] Harris, Ch. \& Stephens, M. (1998). A combined corner and edge detectors, In Alvey Vision Conference, pp. 147-152

[5] Haralick, R. M. \& Shapiro, L. G. (1993). Computer and robot vision, Addison-Wesley, ISBN 0-201-56943-4, 2, pp. 332-347

[6] Lucchese, L. \& Mitra, M. (2002). Using saddle points for subpixel feature detection in camera calibration targets, Circuits and Systems, APPCCAS '02. 2002 Asia-Pacific Conference on, 2, pp. 191-195

[7] Weixing, Z., Changhua, M., Libing, X. \& Xincheng L. (2009). A fast and accurate algorithm for chessboard corner detection, Image and Signal Processing, CISP '09. $2^{\text {nd }}$ International Congress on, pp. 1-5

[8] Chen, D. \& Zhang, G. (2005). A new sub-pixel detector for Ccorners in camera calibration targets, In WSCG (Short Papers), pp. $97-100$

[9] Juhas, M. (2012). Mikropolohovaci stol s krokovym pohonom: technicka sprava, Bratislava: STU, 2012, pp. 1 - 136 